\title{
Rapid-Sequence MRI of the Brain: A Distinct Imaging Study
}

$\mathbf{R}_{b}^{a t}$ apid-sequence MRI of the brain (also known as "ultrafast brain," "quick brain," "fast brain," and "one bang” MRI) has long been used in the evaluation of ventricular shunt catheters due to its ability to quickly evaluate intracranial fluidcontaining spaces without anesthesia or the ionizing radiation of CT. Despite its value, there is no mention of the technique in the American College of Radiology Appropriateness Criteria (https://www.acr.org/Clinical-Resources/ACR-AppropriatenessCriteria), which do treat functional MRI and MR spectroscopy as distinct examinations, likely because these tests have distinct billable procedure codes.

When I moved from one large children's hospital to another during my training, I saw firsthand a distinct difference in the use of rapid-sequence MRI. At my first institution, it was being ordered almost exclusively by neurosurgeons to guide neurosurgical management-specific decisions (shunt malfunction, hydrocephalus, cyst evaluation, and so forth). At my second, however, the technique had far greater reach among the gamut of pediatric specialists who were concerned about the possible neurotoxic effects of anesthesia and wanted to simply "rule out anything big" in patients with suspected neurologic pathology. In a count of 100 consecutive rapid MRI examinations read by a single radiologist from the same starting date at both sites, I found that rapid MRI had only composed about $15 \%$ of the total brain MRI at the restrictive hospital, while the figure was closer to $35 \%$ at the other. The permissive institution had about $8 \%$ of studies ordered for disorders unrelated to CSF-containing spaces (eg, developmental delay, altered mental status, family history of anomalies, and seizures), while the rate was only $3 \%$ at the more restrictive institution. Less than half of the examinations ( $46 \%$ at the restrictive site and $41 \%$ at the permissive site) were performed on children with shunts in place, similar to rates of $35 \%$ and $40 \%$ cited by prior authors. ${ }^{1,2}$ A notable difference in practice was that at the restrictive institution, all sedated MRI was checked by a radiologist before the examination was completed. Both sites partnered with privately contracted anesthesiologists.

Different pediatric specialists have different motivations for ordering rapid MRI. In extra-axial hematoma follow-up, for ex-

http://dx.doi.org/10.3174/ajnr.A5685 ample, a child abuse pediatrician may prefer a sedated conventional MRI, while a neurosurgeon may find rapid MRI suitable, even though this has been shown to be insensitive for evaluating abusive head trauma. ${ }^{3}$ A general pediatrician may be able to get rapid MRI more quickly than conventional MRI; thus, the rapid study becomes a tool to expedite discharge planning. Clinicians may move from one hospital to another without knowing which sequences are included in the study at the new site (eg, diffusionweighted imaging, which makes the examination far more sensitive for ischemia and/or chemotherapy-related toxicity). The sequences used, specific techniques used during image acquisition, and detail of the reports issued have great consequences for patients. Uninformed pediatricians may accept rapid brain MRI "normal” findings as truly normal MRI examinations of the brain. What appears to be an arachnoid cyst on rapid MRI may be the cystic portion of a tumor, which the insensitive nature of the rapid MRI cannot detect.

Imaging centers associated with both institutions referenced above charged for rapid MRI using the same billable procedure code as a conventional MRI of the brain. Neuroradiologists must work to change this practice by designating the rapid-sequence MRI of the brain as a distinct limited study deserving its own charge. Not only is the interpretation of the examination less complex, but the acquisition of the images requires far less equipment-use time. At those centers where a rapid MRI is less expensive than a conventional MRI, how are insurers to know when they should pay for a rapid MRI and deny the order for a conventional MRI, which may incur an additional cost for general anesthesia? With regard to conventional pediatric MRI, what about requests for $3 \mathrm{~T}$ and advanced sequences that require extra cost and training? How are we to decide who deserves these free extra services? Our billing system has simply not kept up with technology.

The appropriate use of rapid-sequence MRI falls somewhere between using it to screen all children with a suspected neurologic abnormality and using it exclusively to evaluate ventricular size. The problem is that without appropriate guidance from the imaging experts that we are, ordering providers are left ignorant of the true sensitivity and specificity of this valuable technique.

\section{REFERENCES}

1. Miller JH, Walkiwewicz T, Towbin RB, et al. Improved delineation of ventricular shunt catheters using fast steady-state gradient recalled-

AJNR Am J Neuroradiol 39:E93-E94 Aug 2018 www.ajnr.org 
echo sequences in a rapid brain MR imaging protocol in nonsedated pediatric patients. AJNR Am J Neuroradiol 2010;31:430-35 CrossRef Medline

2. Rozovsky K, Ventureyra EC, Miller E. Fast-brain MRI in children is quick, without sedation, and radiation-free, but beware of limitations. J Clin Neurosci 2013;20:400-05 CrossRef Medline

3. Kralik SF, Yasrebi M, Supakaul N, et al. Diagnostic performance of ultrafast brain MRI for evaluation of abusive head trauma. AJNR Am J Neuroradiol 2017;38:807-13 CrossRef Medline

(D.M. Pfeifer

Department of Radiology

University of Texas Southwestern Medical Center

Dallas, Texas 